\title{
高速动车组轴箱轴承滚道载荷特性研究*
}

\author{
查 浩 任尊松 徐 宁 \\ (北京交通大学机械与电子控制工程学院 北京 100044)
}

\begin{abstract}
摘要: 轴箱轴承作为列车走行部件中重要核心部件之一, 由于其受载工况恶劣使其成为易损坏的部件之一, 研究其在运行 过程中滚子滚道间的载荷特性对基于载荷分布的轴承寿命预测, 保证其在运用过程中的安全性和可靠性具有重要意义。基 于包含轴承的车辆-轨道动力学模型, 研究了轨道激扰和列车运行速度下滚子滚道接触载荷特征。研究结果表明, 无激扰 条件下, 车速对滚子滚道接触载荷基本没有影响, 但在非承载区, 滚子与外圈滚道会发生接触, 接触载荷与速度成平方关 系; 轨道激扰条件会影响能够引起滚子滚道接触载荷瞬时值发生变化; 外圈受到的接触载荷标准差值, 车速越快, 标准差 越大; 对外圈受到的接触载荷最大的区域进行研究, 发现随着速度的增加, 轨道激扰造成的振动越大, 滚子滚道接触载荷 数据波动越大。
\end{abstract}

关键词: 动车组; 轴箱轴承; 振动加速度; 接触载荷

中图分类号: U271

\section{Load Characteristics of Axle Box Bearing Raceway of High-speed EMU}

\author{
ZHA Hao REN Zunsong XU Ning
}

(School of Mechanical and Electronic Control Engineering, Beijing Jiaotong University, Beijing 100044)

\begin{abstract}
Axle box bearing is an important core department of the train running parts, but it becomes one of the vulnerable parts , because of its bad load condition. It is of great significance for bearing life prediction based on load distribution and ensuring its safety and reliability by studying the load characteristics between roller raceways in operation. Based on the dynamic model of vehicle-track dynamic model with bearings, the contact load characteristics of roller raceway under track excitation and train running speed are studied. The results show that under the condition of no excitation, the speed has little effect on the contact load of the roller raceway, but in the non-bearing area, the roller will contact with the outer raceway, and the contact load is square with the speed. The track excitation condition will affect the instantaneous value of contact load of roller raceway. The standard deviation of the contact load on the outer ring increases with the speed of the vehicle. By studying the maximum contact load on the outer ring, it is found that with the increase of speed, the greater the vibration caused by track excitation, the greater the fluctuation of contact load data of roller raceway.
\end{abstract}

Key words: high speed vehicle; axle box bearing; vibration acceleration; contact load

\section{0 前言}

轴箱轴承作为列车走行部件的核心部件之一, 其关键技术的研究具有重要意义。轴箱轴承在列车 运行的时候不仅需要承受较大的径向载荷, 同时还 需要承受一定的轴向载荷, 属于比较容易损坏的部 件之一 ${ }^{[1]}$, 为了保证其在运用过程中的安全性和可 靠性, 需要研究轴承内部接触载荷的变化情况。

目前, 对高速动车组轴箱轴承滚子滚道间相 互作用研究的文献很多, 颜家森等 ${ }^{[2]}$ 建立了高速

* 国家重点研发计划(2016YFB1200506-21)和国家自然科学基金(11790281) 资助项目。20190622 收到初稿, 20191012 收到修改稿
铁路轴箱轴承双列圆雉滚子轴承的分析模型, 采 用数值分析的方法计算了轴承的载荷分布。王超 ${ }^{[3]}$ 基于 hertz 接触理论与滚子切片理论, 对高铁圆雉 滚子轴承滚子滚道间的接触问题进行研究，求解 了滚子在母线方向上的应力与载荷分布。赵方伟 ${ }^{[4]}$ 根据圆雉滚子轴承力学模型, 计算了高速动车组 列车的载荷分布。郝烨江、郑静等 ${ }^{[5-7]}$ 利用软件 ABAQUS 分析轴承静态接触下的载荷分布与应力 问题。魏延刚等 ${ }^{[8-9]}$ 用有限元法对受力最大滚动体 进行修形设计的精细研究, 在不同工况下分析圆 雉滚子与内、外圈之间的最大接触应力沿其母线 的分布规律。

然而上述研究均没有考虑轴承的动态效应, 
求解载荷分布均是在静态下求解, 无法真是反映 轴承在实际运用状态下滚子与滚道间的相互作用 关系, 鉴于此, 本文基于包含轴承的车辆-轨道动 力学模型, 考虑了轴承内部部件的相互作用, 求 解滚子滚道接触载荷值, 并借助统计特征的均值、 标准差来分析在轨道激扰和不同车速下的滚子滚 道接触载荷特征, 研究结果对揭示轨道激扰下部 件运动规律和高速列车轴承寿命评估具有一定的 指导意义, 也可为轴箱轴承台架试验加载提供一 定参考。

\section{1 轴承-车辆-轨道耦合动力学建模}

本文采用基于包含轴承的车辆-轨道动力学模 型 ${ }^{[10]}$, 各主要部件的运动和约束关系如图 1 所示 ${ }^{[11]}$ 。

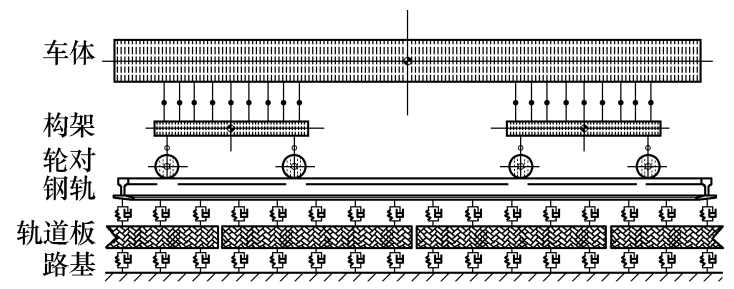

(a) 车辆 - 轨道耦合动力学整体模型

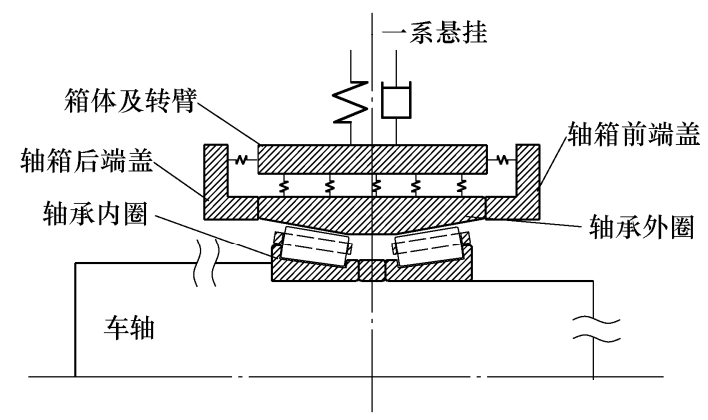

(b) 轴箱局部细节模型

图 1 车辆-轨道耦合动力学模型

模型中, 车辆系统包括车体、2 个构架和 4 个 轮对, 其中车体和构架考虑浮沉、点头、侧滚、摇 头和横移等自由度, 轮对考虑浮沉, 侧滚, 横移和 摇头等自由度。车体与构架之间、构架与轴箱之间 均简化成弹簧-阻尼系统。

轨下部分采用板式无砟轨道模型, 其中钢轨简 化为两端简支的欧拉梁, 轨下扣件约束简化为离散 点支撑刚度和阻尼, 轨道板考虑成下部受连续分布 线性弹簧与线性阻尼支撑的边界自由弹性板 ${ }^{[12]}$ 。轮 轨空间几何接触关系采用轨迹法进行计算 ${ }^{[13]}$ 。

轴箱轴承内部相互作用包含滚子与内外滚道的 相互作用、滚子与内圈大挡边的相互作用、滚动体 与保持架间的相互作用可参照文献[14-16]。则滚子 运动微分方程如下。
切向运动(滚子在滚道向前滚动)

$$
\begin{aligned}
\ddot{\varphi}_{r i j}= & \left(\left(\boldsymbol{Q}_{j(x)}^{o}+\boldsymbol{Q}_{j(x)}^{i}+\boldsymbol{Q}_{j(x)}^{d}+\boldsymbol{Q}_{j(x)}^{c}\right) /\right. \\
& \left.m_{b}+g \sin \varphi_{r i j}-2 \dot{z}_{r i j} \dot{\varphi}_{r i j}\right) / z_{r i j}
\end{aligned}
$$

横移运动

$$
\ddot{y}_{r i j}=\left(\boldsymbol{Q}_{j(y)}^{o}+\boldsymbol{Q}_{j(y)}^{i}+\boldsymbol{Q}_{j(y)}^{d}+\boldsymbol{Q}_{j(y)}^{c}\right) / m_{b}
$$

径向运动

$$
\begin{gathered}
\ddot{z}_{r i j}=\left(\boldsymbol{Q}_{j(z)}^{o}+\boldsymbol{Q}_{j(z)}^{i}+\boldsymbol{Q}_{j(z)}^{d}+\boldsymbol{Q}_{j(z)}^{c}\right) / \\
m_{b}-g \cos \varphi_{r i j}+z_{r i j} \dot{\varphi}_{r i j} \dot{\varphi}_{r i j}
\end{gathered}
$$

侧滚运动

$\ddot{\phi}_{r i j}=\left(\boldsymbol{M}_{j(x)}^{o}+\boldsymbol{M}_{j(x)}^{i}+\boldsymbol{M}_{j(x)}^{d}+\boldsymbol{M}_{j(x)}^{c}\right) / I_{b x z}$

旋转运动

$\ddot{\beta}_{r i j}=\left(\boldsymbol{M}_{j(y)}^{o}+\boldsymbol{M}_{j(y)}^{i}+\boldsymbol{M}_{j(y)}^{d}+\boldsymbol{M}_{j(y)}^{c}\right) / I_{b y}$

摇头运动

$\ddot{\psi}_{r i j}=\left(\boldsymbol{M}_{j(z)}^{o}+\boldsymbol{M}_{j(z)}^{i}+\boldsymbol{M}_{j(z)}^{d}+\boldsymbol{M}_{j(z)}^{c}\right) / I_{b x z}$

式中, $\boldsymbol{Q}$ 表示相互作用力, $\boldsymbol{M}$ 表示相互作用力矩; 上标 $o$ 表示滚子与外圈的相互作用, 上标 $i$ 表示滚 子与内圈的相互作用, 上标 $d$ 表示滚子与内圈挡边 的相互作用，上标 $c$ 表述滚子与保持架的相互作用; 下标 $j$ 表示第 $j$ 个滚子; $m_{b}$ 表示滚子质量; $I_{b y}$ 表示 滚子过质心绕自身轴线的转动惯量; $I_{b x z}$, 表示滚子 过质心在垂直自身轴线的转动惯量。

\section{2 滚子-滚道接触载荷时域特征分析}

\section{1 基于动力学模型无激扰条件下滚子滚道接触 载荷特征}

目前求解轴承载荷分布的方法主要有两种，分 别是理论计算求解和载荷分布数值计算模型 ${ }^{[2]}$ 求 解, 理论计算在求解过程中需要通过查图来获得载 荷分布系数 $\varepsilon$ 下载荷积分 $J_{\mathrm{r}}$ 的值, 所以采用载荷分 布数值计算模型更为简便一些。但传统的载荷分布 计算是基于轴承的静态模型求解的，无法考虑滚子 的运动特征带来的力。

在使用静态模型求解载荷分布的时候，先获取 滚子对应滚道位置的载荷值, 再用曲线拟合, 即为 载荷分布图, 运用载荷分布图可以得知滚子在滚道 任意位置时滚子滚道接触载荷值; 而在动力学模型 求解时, 可以直接输出滚子与滚道在任意位置时的 接触载荷。

无激扰条件下列车不同运行速度时单个滚子与 内圈接触载荷及其放大图如图 2 所示。 


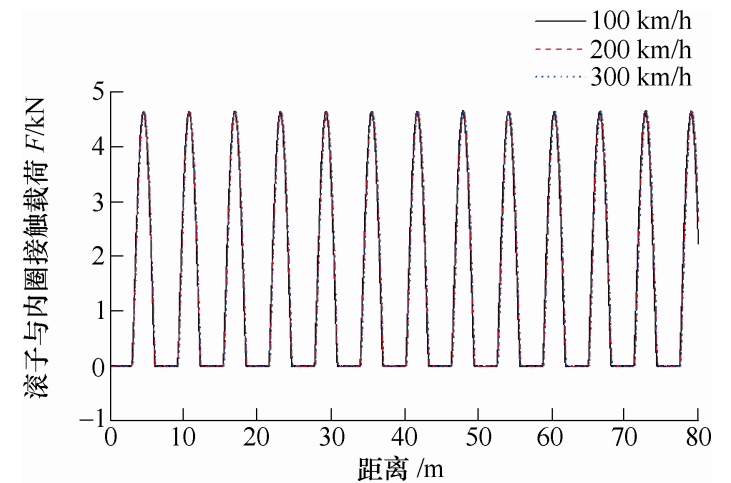

(a) 整体结果

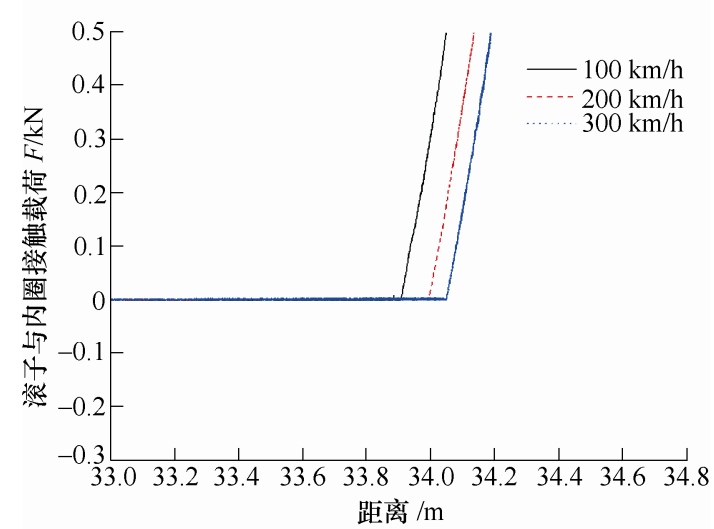

(b) 局部放大结果

图 2 滚子与内圈滚道接触载荷时间历程

由图 2a 可知, 在没有轨道激扰条件下, 不同速 度级下的滚子滚道接触载荷均呈现周期性的变化, 且最大最小值几乎无差别, 由于在非承载区滚子与 内圈滚道没有接触, 所以接触载荷为 0 ; 由局部放 大图 $2 b$ 显示, 不同速度级下的结果具有一定的相位 差, 即速度越慢, 越早出现峰值, 这种相位差与系 统内部的打滑有关, 所以在无激扰条件下, 速度对 滚子滚道接触载荷的周期性、幅值大小均没有影响, 但对结果的相位有影响。需要说明的是, 局部图中 数据结果的微小高频振动对应的频率为轴承运动带 来的振动频率及其倍频, 后续文中还会具有类似的 现象出现, 不再赘述。

无激扰条件下列车不同运行速度时单个滚子与 外圈接触载荷及其放大图如图 3 所示。

在整体趋势上, 滚子-内圈滚道接触载荷与滚子外圈滚道接触载荷一致, 但在非承载区域, 局部放大 图 $3 \mathrm{~b}$ 显示, 滚子由于重力作用滚子和外圈滚道会发 生接触, 且在整个非承载区域内不同速度级下，所有 数值均远大于滚子自身的重力, 由图 3 可知 $100 \mathrm{~km} / \mathrm{h}$ 速度下, 非承载区接触载荷数据在 $4.2 \mathrm{~N}$ 附近波动; $200 \mathrm{~km} / \mathrm{h}$ 速度下, 非承载区接触载荷数据在 $16.7 \mathrm{~N}$ 附近波动, 为 $100 \mathrm{~km} / \mathrm{h}$ 下的结果的 3.98 倍; $300 \mathrm{~km} / \mathrm{h}$ 速度下, 非承载区接触载荷数据在 $37.6 \mathrm{~N}$ 附近波动, 为 $100 \mathrm{~km} / \mathrm{h}$ 下的结果的 8.95 倍。即 $100 \mathrm{~km} / \mathrm{h}$ ：

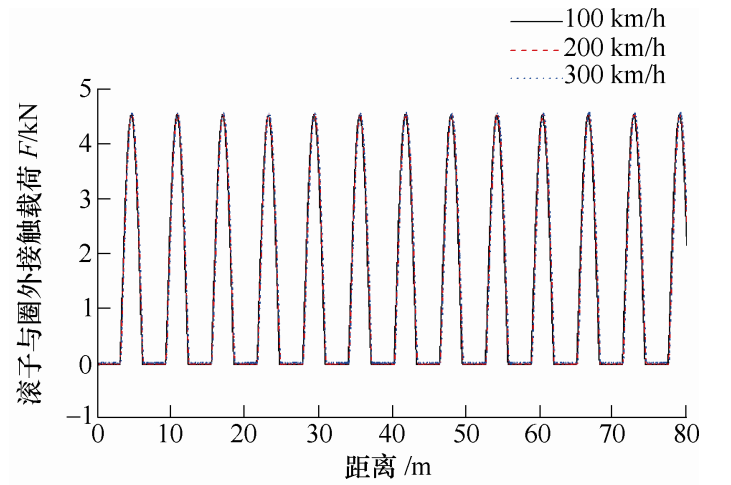

(a) 整体结果

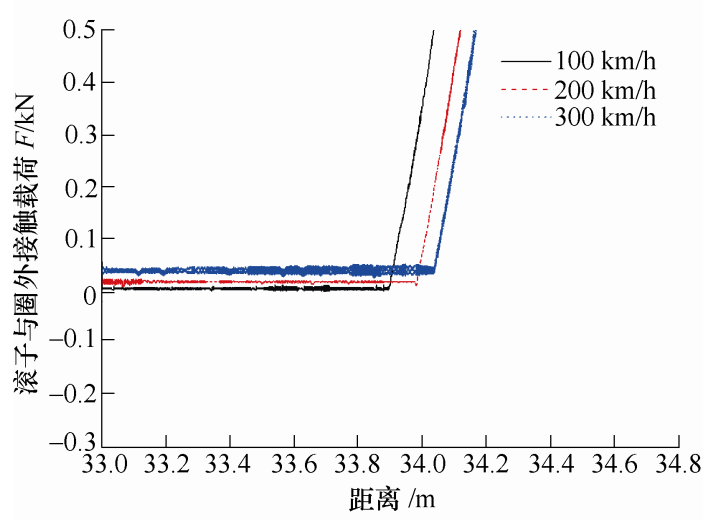

(b) 局部放大结果

图 3 滚子与外圈滚道接触载荷时间历程

$200 \mathrm{~km} / \mathrm{h}: 300 \mathrm{~km} / \mathrm{h}$ 的结果=1 $: 3.98: 8.95$, 近似为 $1: 2^{2}: 3^{2}$, 呈现平方递增的关系, 说明非承载区 的滚子-外圈的接触载荷值与列车运行的速度有 关, 这是传统理论计算和静态载荷分布数值计算 模型所无法体现的。

将外圈滚道进行区域划分, 如图 4 所示, 最顶 端定义为 0 区域，按照滚子转动的方向按顺序正编 号, 滚子转动的逆方向按顺序负编号。

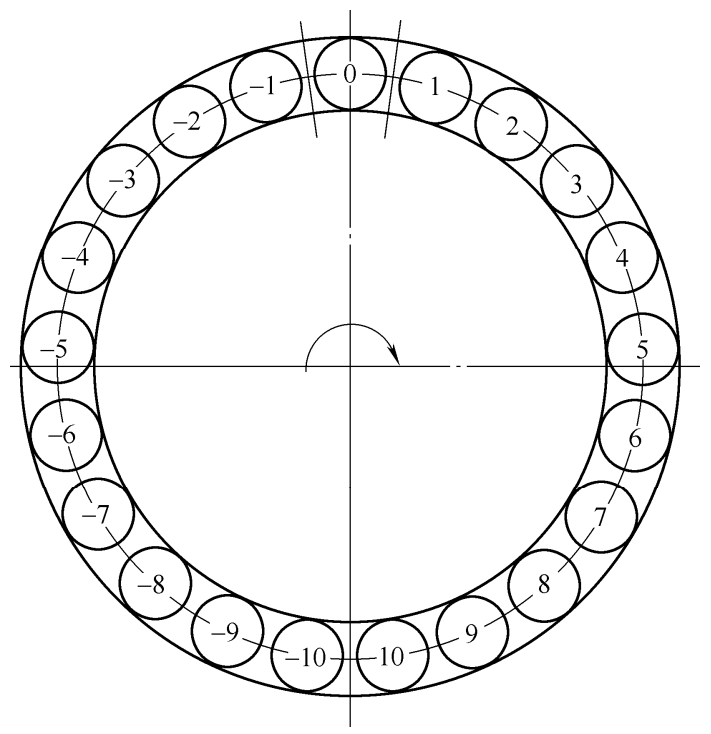

图 4 外圈滚道区域划分

根据图 3 滚子与外圈滚道接触载荷时间历程图 
绘制滚道划分下滚子与外圈滚道接触载荷分布图, 如图 5 所示, 并与静力学模型 ${ }^{[2]}$ 求解结果进行比对。

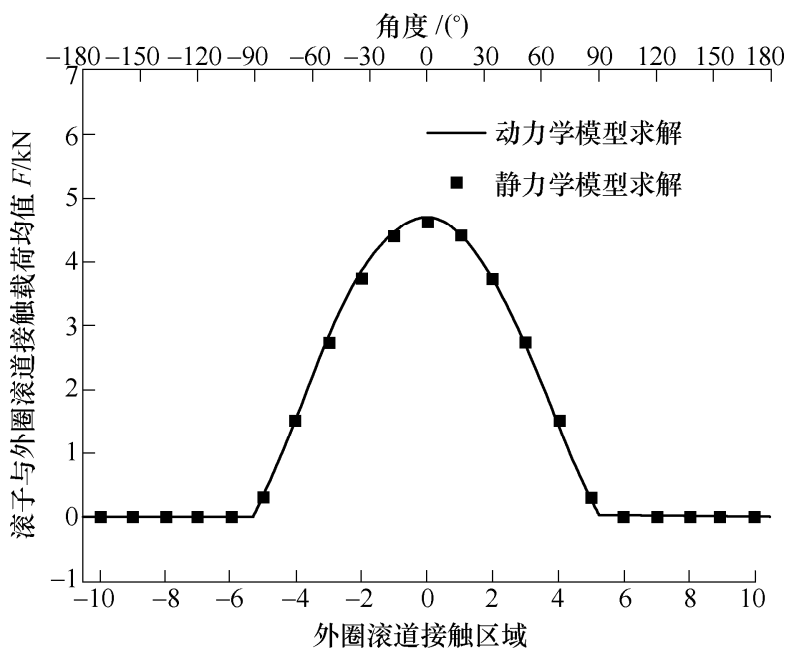

图 5 滚子与外圈滚道接触载荷分布图

由图 5 可知, 滚子与外圈滚道接触载荷分布图 与静力学模型求解的载荷分布图一致。

\section{2 激扰条件下滚子滚道接触载荷特征}

轨道激扰采用实测 “京津谱” 轨道激扰, 如 图 6 所示。

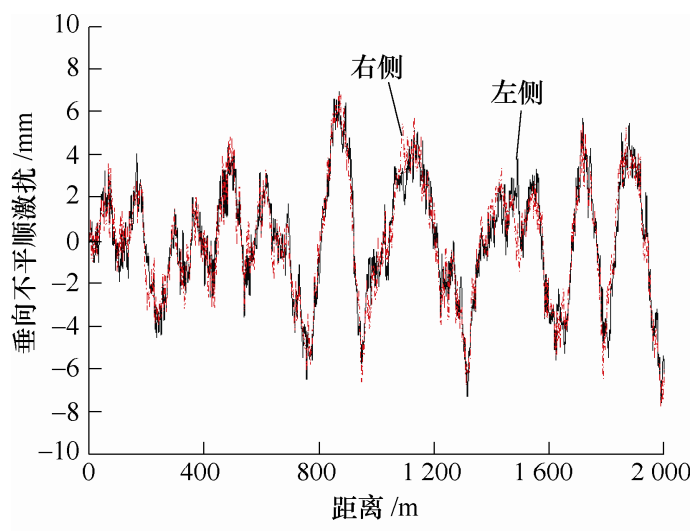

(a) 垂向不平顺

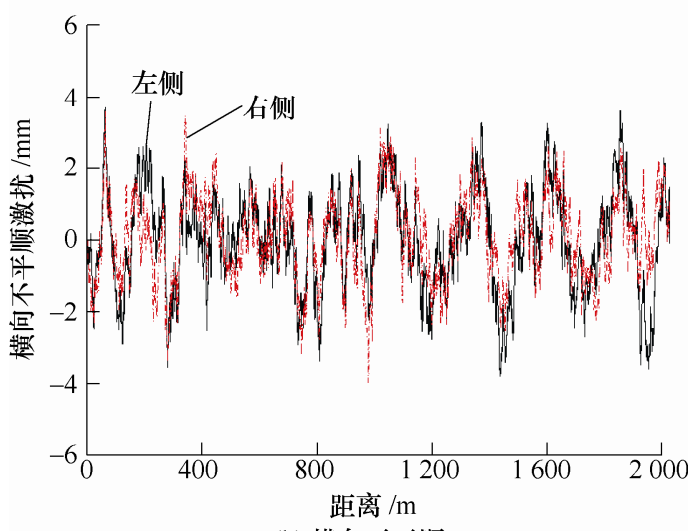

(b) 横向不平顺

图 6 “京津谱” 轨道谱激扰

轨道激扰条件下, 列车不同运行速度时单个滚 子与外圈接触载荷如图 7 所示。

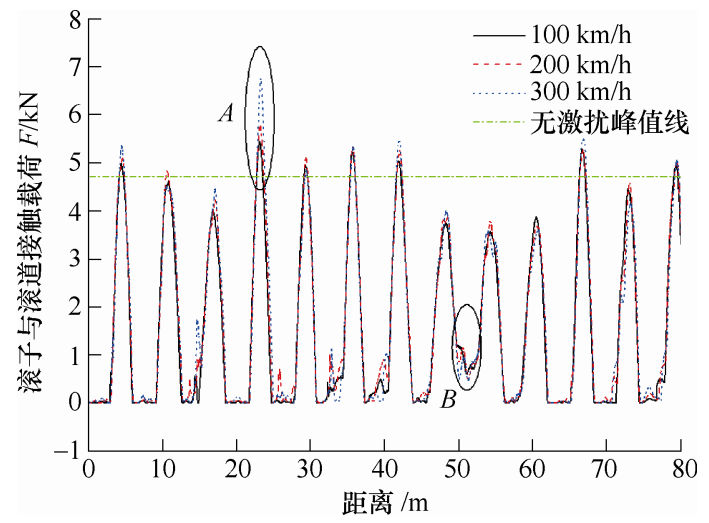

图 7 滚子与外圈滚道接触载荷时间历程

由图 7 可知, 在轨道激扰条件下, 非承载区中 滚子与外圈发生了接触碰撞, 并会影响滚子-滚道间 接触载荷的峰值大小, 在 $300 \mathrm{~km} / \mathrm{h}$ 的速度下, 最大 峰值能够达到 $6.75 \mathrm{kN}$, 约为无激扰条件下的 1.5 倍。 图中无激扰峰值线由图 3a 获得。

给出图 8 中 $A$ 和 $B$ 处在 $300 \mathrm{~km} / \mathrm{h}$ 下同一轴承第 一列(外侧)和第二列(内侧)滚子与外圈滚道的瞬时 接触载荷, 如图 7 所示。

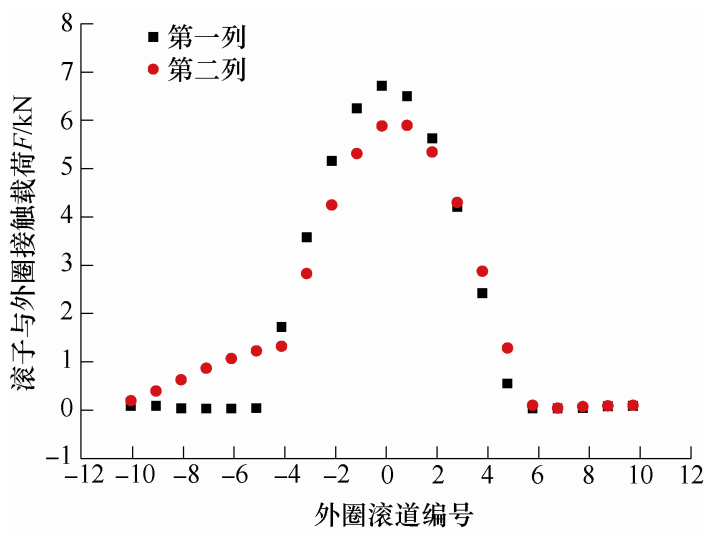

(a) 点 $A$ 瞬时

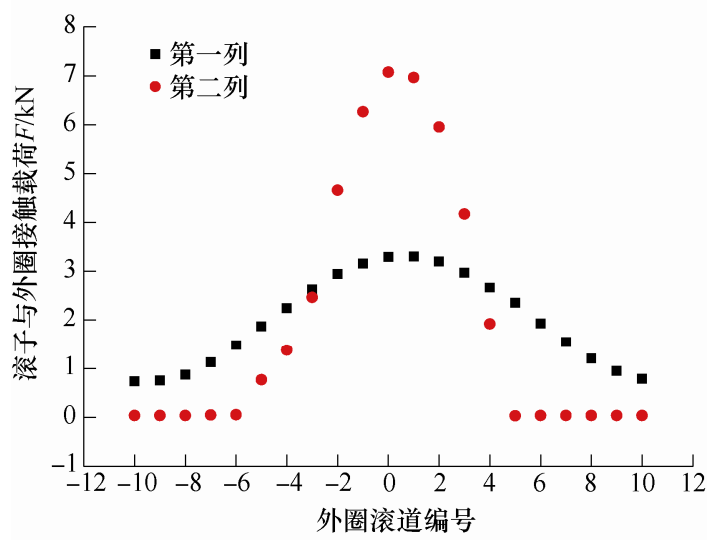

(b) 点 $B$ 瞬时

图 8 滚子与外圈滚道瞬时接触载荷

由图 8 可知, 在 $A$ 时刻, 第一列外圈滚道最大 值为 $6.71 \mathrm{kN}$, 受载滚子数为 10 个, 非受载滚子为 11 个, 第二列外圈滚道最大值为 $5.89 \mathrm{kN}$, 最大值 并没有出现在滚道最上端 0 区域, 而是出现在了 1 
区域, 且滚子的承载数增加; 在 $B$ 时刻第一列外圈 滚道全部受载, 第二列外圈滚道最大值为 $7.07 \mathrm{kN}$, 是第一列外圈滚道最大值的 2 倍, 且没有承载的滚 子数为 11 。造成左右列分布差异的原因是由于在运 动过程中, 轴承内圈相对外圈运动导致的。

综上来看, 在轨道激扰下, 滚子与外圈滚道接 触载荷时刻变化, 故无法按照滚道划分来绘制激扰 下滚子与外圈滚道接触载荷分布图。

滚子与外圈滚道的瞬时值只能反映某一时刻滚 子滚道的运动状态, 且单一滚子与滚道接触载荷无 法反映激扰条件下所有滚子滚道的载荷接触特征, 可以借助统计学方法, 统计外圈滚道按区域划分后, 每个区域受所有滚子接触载荷的影响。

\section{3 滚子-滚道接触载荷统计特征分析}

\section{1 激扰条件下外圈滚道接触载荷均值特征}

统计有无激扰条件下, 各个区域受所有滚子接 触载荷的均值分布图, 如图 9 所示。

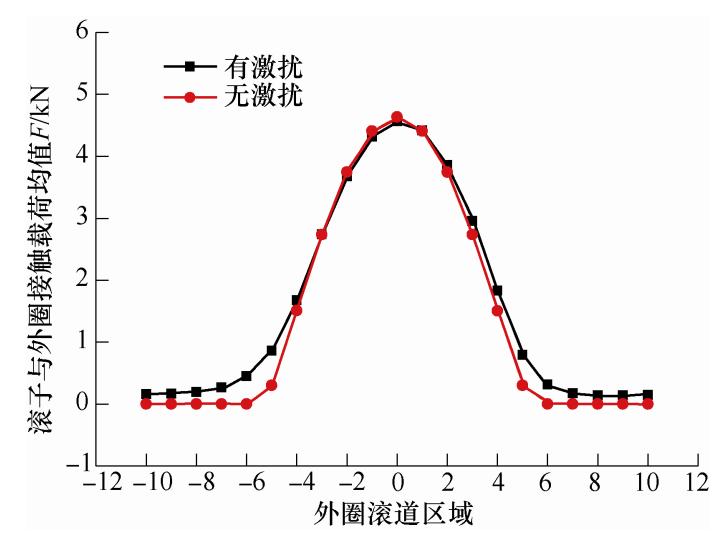

图 9 滚子与外圈各个区域内接触载荷均值分布图

由图 9 可知, 在无激扰条件下, 滚子与外圈滚 道在 “ 0 ” 区域内接触载荷均值为 $4.64 \mathrm{kN}$, 略小于 图 3a 中滚子-外圈滚道接触载荷最大值为 $4.72 \mathrm{kN}$, 且图形在横坐标 0 处呈现左右对称。在轨道激扰条 件下, 图形在横坐标 0 处不再呈现左右对称, 在承 载区域内, 编号为正的区域的均值均大于编号为负 的区域的均值, 即结果 $1>-1,2>-2$; 而在非承载 区内, 编号为正的区域的均值均小于编号为负的区 域的均值, 即结果 $10<-10,9<-9$ 。对比有无轨道 激扰条件下的结果可知, 在 0 及其靠近区域, 无激 扰的接触载荷均值结果略大于有激扰的结果, 但在 非承载区域内, 有激扰的接触载荷均值结果大于无 激扰的结果。

统计同一轴承第一列(外侧)和第二列(内侧)滚 子与外圈各个区域内的接触载荷均值分布对比如
图 10 所示。

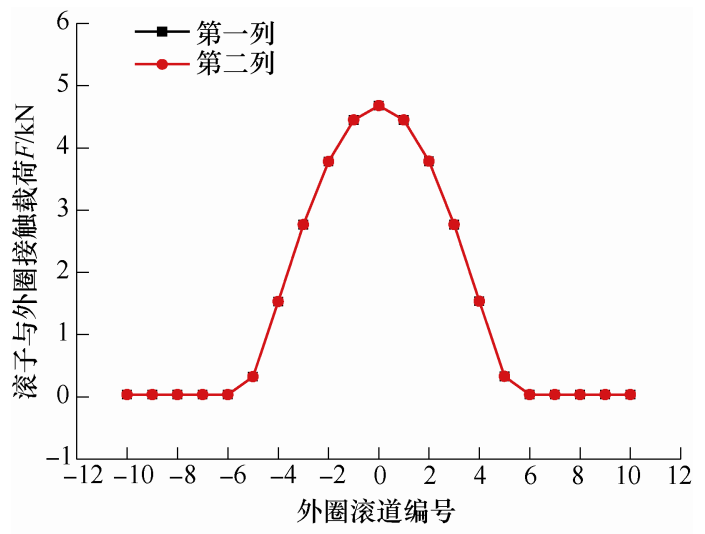

(a) 无激扰

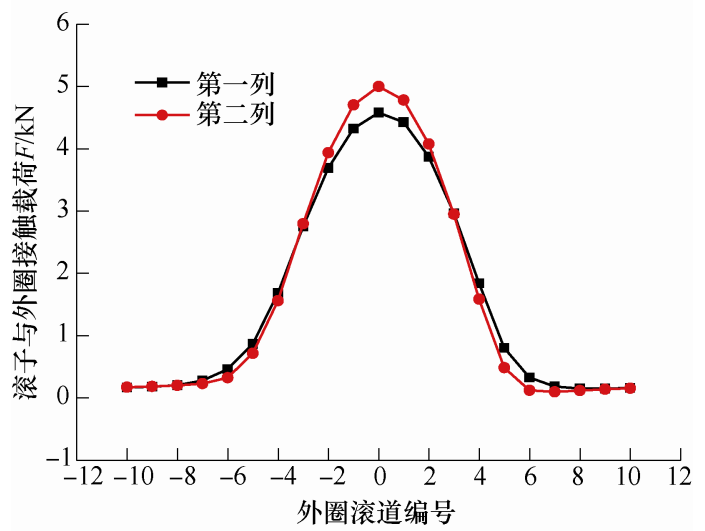

(b) 有激扰

图 10 两列滚子与外圈各个区域内的接触载荷均值对比图

由图 10 可知, 在无激扰条件下, 两列滚子与 外圈各个区域内的接触载荷均值外圈完全相同, 但 在有轨道激扰条件下, 在承载区, 第一列<第二列, 在非承载区, 第一列>第二列, 说明在这段轨道激 扰下, 有横向力使得内圈相对外圈在轴向位置发生 位移，导致第一列轴向间隙增大，而第二列轴向间 隙减小, 这一结果与轴箱对应位置的一系力结果对 应(图 11), 这段区域内一系力横向力均值为 $36 \mathrm{~N}$ 。

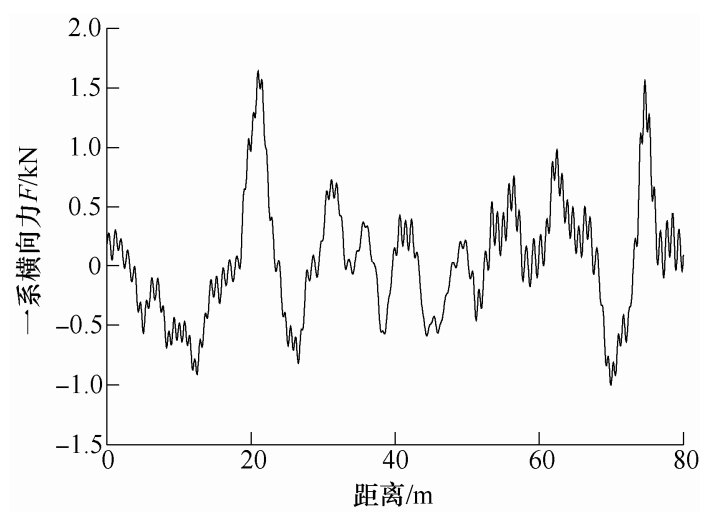

图 11 一系横向力

图 12 给出了激扰条件下, 不同车速等级下的滚 子与外圈各个区域内接触载荷均值。 


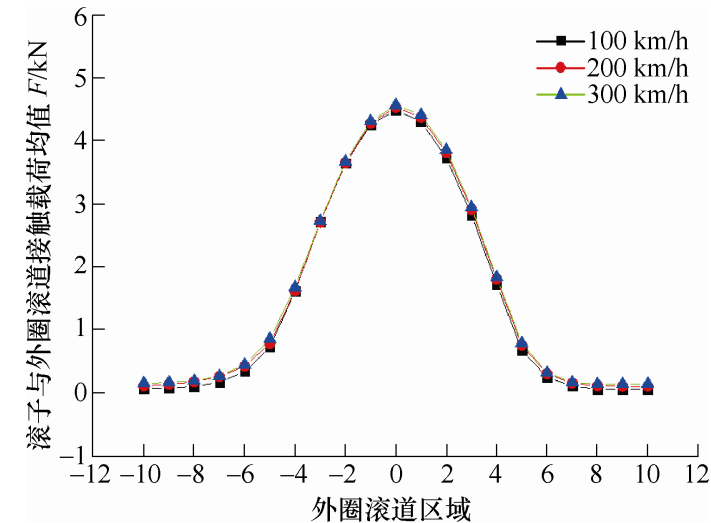

图 12 滚子与外圈各个区域内接触载荷均值分布图

由图 12 可知, 滚子与外圈各个区域内接触载荷 均值在不同速度级下总体趋势一致, 且差距不是很 明显。

综上来看, 高速列车轴箱轴承滚子与外圈滚道 载荷统计均值与无激扰恒定载荷下结果差距不大, 但轨道随机激扰具有离散型特征, 势必使滚子与外 圈滚道载荷的具有离散特征, 可以用标准差来衡量 这一离散特征。

\section{2 激扰条件下外圈滚道接触载荷离散特征}

图 13 给出了车速 $300 \mathrm{~km} / \mathrm{h}$, 滚子与外圈各个区 域内接触载荷标准差分布图。

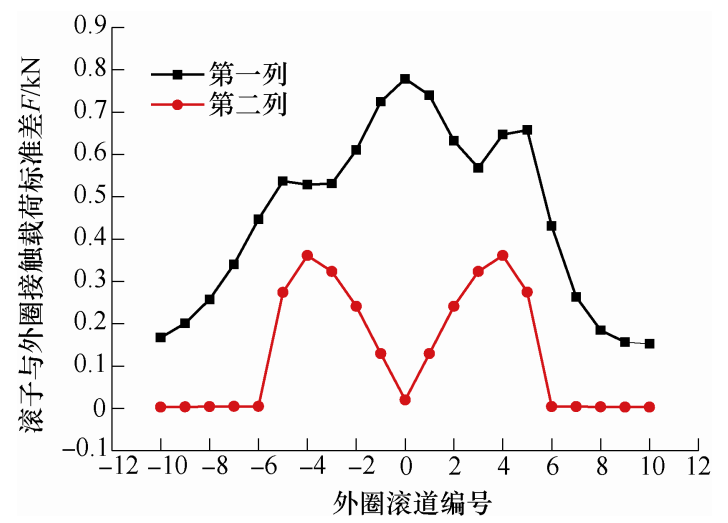

图 13 滚子与外圈各个区域内接触载荷标准差分布图

由图 13 可以看出, 在无激扰情况下, 部分区域 标准差接近为 0 , 几乎没有波动, 其中载荷均值最 大的 0 区域, 标准差很小, 为 $0.02 \mathrm{kN}$ 。在轨道激扰 条件下, 所有区域标准差明显增大, 其中 0 区域标 准差值最大, 且相较于无激扰下的标准差值, 0 区 域也是标准差值变化最明显的区域, 说明轨道激扰 对该区域载荷的影响最为明显。

图 14 给出了不同车速下, 滚子与外圈各个区域 内接触载荷标准差分布图。

由图 14 可知, 在外圈滚道的区域内, 在相同的 轨道激扰下, 随着速度的增加, 滚子与外圈接触载 荷标准差越大, 说明轨道激扰对外圈接触载荷的影 响不可忽略。

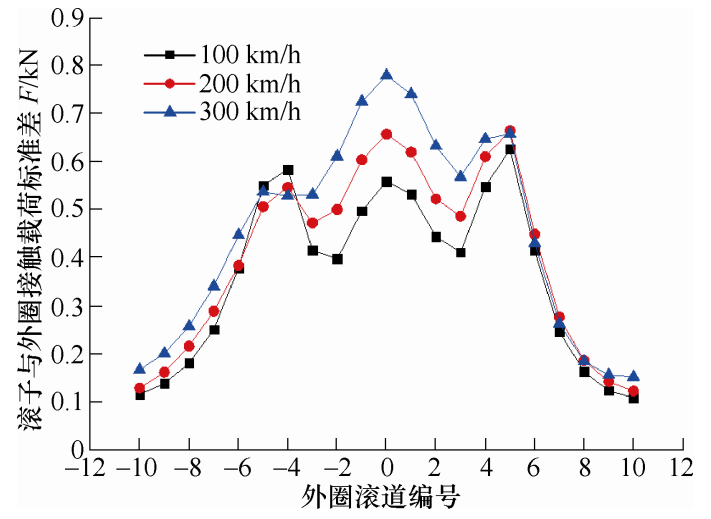

图 14 滚子与外圈各个区域内接触载荷标准差分布图

\section{3 单一滚道区域滚子外圈接触载荷特征}

为进一步说明轨道激扰和列车运行速度对外 圈滚道接触载荷的影响, 选取滚子外圈滚道接触载 荷均值最大、标准差变化最大的滚道 0 区域进行研 究, 图 15 给出 0 区域的滚子外圈滚道接触载荷时 间历程。

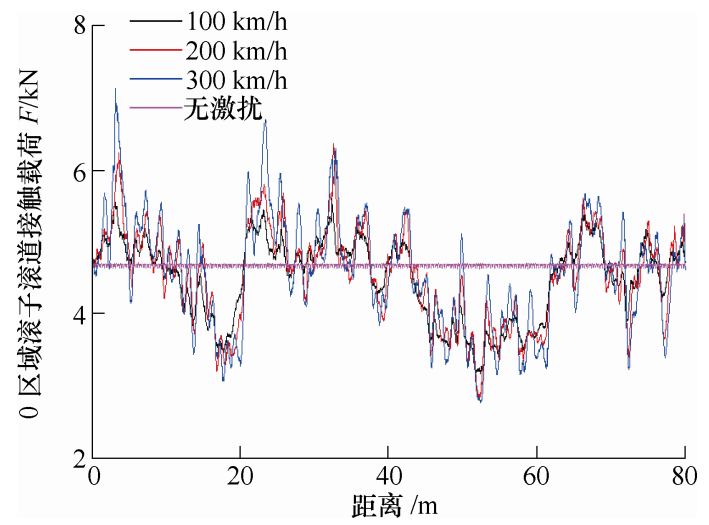

图 15 滚子与外圈在 0 区域接触载荷历程

由图 15 可以看出, 无激扰下滚子滚道接触载荷 稳定在 $4.64 \mathrm{kN}$ 附近且波动微小，这种微小波动来 自于滚子在滚道中位置的变化; 在有激扰的情况下， 滚子滚道接触载荷波动十分明显, 相较于无激扰载 荷, 最大波动幅值为 $2.5 \mathrm{kN}$ 。为了考虑幅值的分布, 将图中所有数据减去对应无激扰数据后取绝对值, 并统计数据落在对应区域的占比, 波动幅值划分为 $0 \sim 0.25,0.25 \sim 0.5, \cdots, 2.0 \sim 2.25,2.25 \sim 2.5$ 共计 10 个区域, 统计结果如图 16 所示。

由图 16 可知, 在所有速度级下, 数据都是围绕 无激扰值 $4.64 \mathrm{kN}$ 波动, 且越靠近中心，数据越集 中。比较不同速度下数据的差异, 在 $0 \sim 0.25,0.25 \sim$ 0.50 区域内, $100 \mathrm{~km} / \mathrm{h}$ 占比为 0.61 大于 $200 \mathrm{~km} / \mathrm{h}$ 的占比 0.53 和 $300 \mathrm{~km} / \mathrm{h}$ 的占比 0.47 ; 在 $0.50 \sim 0.75$, $0.75 \sim 1.0$ 区域内, $200 \mathrm{~km} / \mathrm{h}$ 占比为 0.32 大于 100 $\mathrm{km} / \mathrm{h}$ 的占比 0.29 和 $300 \mathrm{~km} / \mathrm{h}$ 的占比 0.31 ; 在剩下 的区域内, $300 \mathrm{~km} / \mathrm{h}$ 占比为 0.22 大于 $100 \mathrm{~km} / \mathrm{h}$ 的 占比 0.10 和 $200 \mathrm{~km} / \mathrm{h}$ 的占比 0.15 。这说明随着速度 


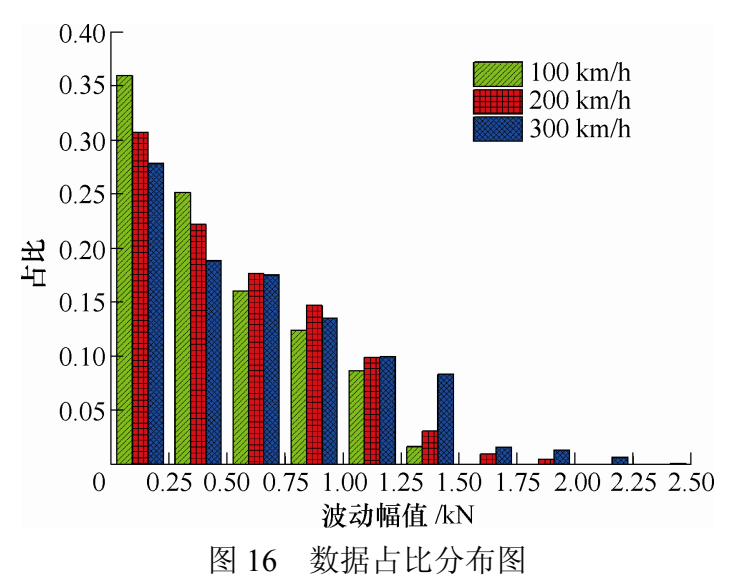

的增加, 轨道激扰造成的振动越大, 滚子滚道接触 载荷数据波动越大。

\section{4 结论}

（1）基于包含轴承的车辆-轨道动力学模型, 考 虑了轴承内部部件的相互作用, 研究了轨道激扰条 件下轴箱轴承滚子-滚道接触载荷特性, 能反映轴 承在耦合大系统中的动态特性, 包括传统模型不能 体现的非承载区的滚子的承载特点, 对基于载荷分 布特征的高速列车轴承寿命评估具有一定的指导 意义。

（2）无激扰条件下，车速对滚子滚道接触载荷 基本没有影响, 且在非承载区, 滚子与外圈滚道会 发生接触, 接触载荷与速度成平方关系。

(3) 轨道激扰条件会影响能够引起滚子滚道接 触载荷瞬时值发生变化, 但统计外圈受到的接触载 荷均值与无激扰条件下对应的均值差异不大, 且不 能反映车速对滚子滚道接触载荷值的影响。

（4）统计外圈受到的接触载荷标准差值, 并与 无激扰条件下对应的标准差值对比, 两者具有明显 差异, 且车速越快, 标准差越大。

（5）对外圈受到的接触载荷均值最大、标准差 变化最大的滚道 0 区域进行研究, 发现随着速度的 增加, 轨道激扰造成的振动越大, 滚子滚道接触载 荷数据波动越大。

\section{参 考 文 献}

[1] 王风才, 王路顺. 高速铁路轴承重大产品工程[R]. 大 连: 国家大型轴承工程技术研究中心, 2011.

WANG Fengcai, WANG Lushun. High speed railway bearing major product engineering[R]. Dalian: National Centre of Bearing Technology, 2011.

[2] 颜家森, 朱龙泉, 赵三星, 等. 高速铁路轴箱轴承载荷 分布分析 [J]. 轴承, 2012(10): 12-16.

YAN Jiasen, ZHU Longquan, ZHAO Sanxing, et al.
Analysis on load distribution of high-speed railway axle box bearings[J]. Bearing, 2012(10): 12-16.

[3] 王超. 高铁圆雉滚子轴承滚子与滚道间的接触分析 [J]. 北京交通大学学报, 2017, 41(4): 91-97.

WANG Chao. Contact analysis between roller and raceway of tapered roller bearing of high-speed rail[J]. Journal of Beijing Jiaotong University, 2017, 41(4): 91-97.

[4] 赵方伟. 高速动车组轴箱轴承接触模型的建立与分析 [J]. 轴承, 2019(3): 1-6.

ZHAO Fangwei. Establishment and analysis on contact model of axle box bearings for high speed EMU[J]. Bearing, 2019(3): 1-6.

[5] 郝烨江, 李强, 郑静. 基于 ABAQUS 的列车轴箱轴承 动力学分析 $[\mathrm{J}]$. 轴承, 2014(3): 10-15.

HAO Yejiang, LI Qiang, ZHENG Jing. Dynamics analysis of train axle box bearings based on ABAQUS[J]. Bearing, 2014(3): 10-15.

[6] 郝烨江. 高速列车轴箱轴承可靠性建模研究[D]. 北京: 北京交通大学, 2014.

HAO Yejiang. Reliability moding and research of the axle box roller bearing used in high-speed train[D]. Beijing: Beijing Jiaotong University, 2014.

[7] 郑静. 高速列车轴箱轴承疲劳寿命研究[D]. 北京: 北京 交通大学, 2014.

ZHENG Jing. Study on the fatigue life of the axle box bearing for high speed train[D]. Beijing: Beijing Jiaotong University, 2014.

[8] 魏延刚, 许凯, 董超. 高速列车轴箱圆锥滚子轴承滚动 体的对称修形 $[J]$. 大连交通大学学报, 2016，37(3): 44-48.

WEI Yangang, XU Kai, DONG Chao. Research on symmetrical modification of tapered roller bearing of high-speed train journal box[J]. Journal of Dalian Jiaotong University, 2016, 37(3): 44-48.

[9] 魏延刚, 董超. 高速列车轴箱圆雉滚子轴承滚子的对数 修形 $[J]$. 大连交通大学学报, 2015, 36(4): 120.

WEI Yangang, DONG Chao. Study on the logarithmic crowned roller of tapered roller bearing of the high-speed train journal box[J]. Journal of Dalian Jiaotong University, 2015, 36(4): 120.

[10] 查浩, 任尊松, 徐宁. 高速动车组轴箱轴承振动特性 [J]. 机械工程学报，2018，54(16): 144-151.

ZHA Hao, REN Zunsong, XU Ning. Vibration performance of high-speed vehicles with axle box bearing[J]. Journal of Mechanical Engineering, 2018, 54(16): 144-151.

[11] REN Zunsong. An investigation on wheel/rail impact dynamics with a three-dimensional flat model[J]. Vehicle 
system dynamics, 2019, 57(3): 369-388.

[12] 翟婉明. 车辆-轨道耦合动力学 [M]. 北京: 科学出版社, 2014.

ZHAI Wanming. Vehicle-track coupling dynamics[M]. Beijing: Science Press, 2014.

[13] 王开文. 车轮接触点迹线及轮轨接触几何参数的计算 [J]. 西南交通大学学报, 1984(1): 89-99.

WANG Kaiwen. The track of wheel contact points and the calculation of wheel/rail geometric contact parameters[J]. Journal of southwest jiaotong university, 1984(1): 89-99.

[14] GUPTAPK. Advance dynamic of rolling elements [M]. New York: Springer-Verlag, 1984.

[15] 胡广存, 魏铁建, 邓四二, 等. 双列圆雉滚子轴承动力 学分析 [J]. 河南科技大学学报, 2014, 35(6): 14-19, 5 . HU Guangcun, WEI Tiejian, DENG Sier, et al. Dynamic analysis of double row tapered roller bearings[J]. Journal of Henan University of Science and Technology, 2014, 35(6): 14-19, 5.

[16] 胡浪, 王文中, 赵自强, 等. 双列圆雉滚子轴承滚子大 端-引导边润滑接触分析[J]. 摩擦学学报, 2013, 33(1): 22-28.

HU Lang, WANG Wenzhong, ZHAO Ziqiang, et al. Lubricated contact analysis of roller large edn-flange in double-row tapered roller bearing[J]. Tribology, 2013, 33(1): $22-28$.

作者简介: 查浩(通信作者), 男, 1991 年出生, 博士研究生。主要研究 方向为车辆系统动力学。

E-mail: 14116362@bjtu.edu.cn

任尊松, 男, 1969 年出生, 博士, 教授, 博士研究生导师。主要研究方 向为车辆系统动力学与结构强度。 